\title{
Philosophiques
}

\section{Droits humains et conflits armés}

\section{Jean-Baptiste Jeangène Vilmer}

Volume 42, numéro 2, automne 2015

Dossier. Les droits humains - nouveaux développements

URI : https://id.erudit.org/iderudit/1034743ar

DOI : https://doi.org/10.7202/1034743ar

Aller au sommaire du numéro

\section{Éditeur(s)}

Société de philosophie du Québec

\section{ISSN}

0316-2923 (imprimé)

1492-1391 (numérique)

Découvrir la revue

\section{Citer cet article}

Jeangène Vilmer, J.-B. (2015). Droits humains et conflits armés. Philosophiques, 42(2), 311-333. https://doi.org/10.7202/1034743ar

\section{Résumé de l'article}

À première vue, il s'agit d'une chose et son contraire : la guerre est tellement le lieu par excellence de la violation des droits humains que leur relation semble se résumer à cet antagonisme primaire - l'un serait la négation de l'autre. La guerre viole les droits et les droits ont la paix, donc l'absence de guerre, comme condition de possibilité. Puis l'on se souvient que, contrairement aux apparences, la guerre n'est pas cet état de non-droit où tout est permis, mais un espace normé, codifié. Il est question des droits humains pendant la guerre pour dénoncer leur violation, certes, mais la violation des règles n'est pas la preuve de leur absence - mais aussi avant la guerre, puisque certains conflits sont justifiés par la protection des droits des populations locales, ou en vertu d'un « droit de l'humanité » qui serait un intérêt à agir. Les relations entre droits humains et conflits armés sont résumées dans cet article en quatre parties : d'un point de vue historique, d'abord, où l'on montre leur réciprocité (le rôle de la guerre dans l'évolution des droits humains, et le rôle des droits humains dans l'évolution de la guerre). En reprenant la trilogie de l'éthique de la guerre ensuite : la guerre au nom des droits humains (jus ad bellum), les droits humains dans la guerre (jus in bello) et, en guise de conclusion, les droits humains après la guerre (jus post bellum).
Ce document est protégé par la loi sur le droit d'auteur. L’utilisation des services d’Érudit (y compris la reproduction) est assujettie à sa politique d'utilisation que vous pouvez consulter en ligne.

https://apropos.erudit.org/fr/usagers/politique-dutilisation/ 


\title{
Droits humains et conflits armés
}

\author{
JEAN-BAPTISTE JEANGĖNE VILMER \\ Sciences Po, Paris \\ jeanbaptiste.jeangenevilmer@sciencespo.fr
}

\begin{abstract}
RÉSUMÉ. - À première vue, il s'agit d'une chose et son contraire: la guerre est tellement le lieu par excellence de la violation des droits humains que leur relation semble se résumer à cet antagonisme primaire - l'un serait la négation de l'autre. La guerre viole les droits et les droits ont la paix, donc l'absence de guerre, comme condition de possibilité. Puis l'on se souvient que, contrairement aux apparences, la guerre n'est pas cet état de non-droit où tout est permis, mais un espace normé, codifié. Il est question des droits humains pendant la guerre - pour dénoncer leur violation, certes, mais la violation des règles n'est pas la preuve de leur absence - mais aussi avant la guerre, puisque certains conflits sont justifiés par la protection des droits des populations locales, ou en vertu d'un «droit de l'humanité » qui serait un intérêt à agir. Les relations entre droits humains et conflits armés sont résumées dans cet article en quatre parties: d'un point de vue historique, d'abord, où l'on montre leur réciprocité (le rôle de la guerre dans l'évolution des droits humains, et le rôle des droits humains dans l'évolution de la guerre). En reprenant la trilogie de l'éthique de la guerre ensuite: la guerre au nom des droits humains (jus ad bellum), les droits humains dans la guerre (jus in bello) et, en guise de conclusion, les droits humains après la guerre (jus post bellum).
\end{abstract}

ABSTRACT. - At first glance these two terms may seem contradictory. War is the most extreme context of human rights violations, suggesting that they have a fundamentally antagonistic relationship and that one is the negation of the other. War violates rights, while rights require peace and hence the absence of war. Despite this appearance, however, war is not a state of lawlessness where all is permitted, but rather a regulated, codified space. There is a question of human rights in war and we obviously denounce their violation, but the violation of rules is not proof of their absence. There is another question of human rights before war, since certain conflicts are justified by the protection of the local population's rights, or the "rights of humanity" as a cause for action. This article summarises the relations between human rights and armed conflicts in four sections: firstly from a historical perspective, focusing on their reciprocity (war's role in the evolution of human rights and the role of human rights in the evolution of war). The next sections follow the trinity of the ethics of war: war in the name of human rights (jus ad bellum), human rights in war (jus in bello) and, to conclude, post-war human rights (jus post bellum).

À première vue, il s'agit d'une chose et son contraire: la guerre est tellement le lieu par excellence de la violation des droits humains que leur relation semble se résumer à cet antagonisme primaire - l'un serait la négation de l'autre. La guerre viole les droits et les droits ont la paix, donc l'absence de guerre, comme condition de possibilité. Puis l'on se souvient que, contrairement aux apparences, la guerre n'est pas cet état de non-droit où tout est 
permis, mais un espace normé, codifié. Il est question des droits humains pendant la guerre - pour dénoncer leur violation, certes, mais la violation des règles n'est pas la preuve de leur inexistence - mais aussi avant la guerre, puisque certains conflits sont justifiés par la protection des droits des populations locales, ou en vertu d'un "droit de l'humanité » qui serait un intérêt à agir.

Leur relation est en réalité tellement riche qu'elle correspond à un domaine de recherche à part entière, l'éthique de la guerre, qui pose la question de sa légitimité: à quelles conditions peut-on dire d'une guerre qu'elle est juste au sens du jus ad bellum (le droit d'entrer en guerre), du jus in bello (le droit dans la guerre) et du jus post bellum (le droit après la guerre)? Les deux premiers datent de la scolastique médiévale, le troisième est plus récent. Kant avait déjà identifié «le problème, d'une part, du droit à la guerre, d'autre part du droit s'appliquant durant la guerre, enfin du droit [...] qui s'instaure après la guerre ${ }^{1}$ ", mais le jus post bellum, sous cette appellation, ne sera développé qu'au début des années $2000^{2}$.

L'éthique de la guerre, qui n'est autre que le nom moderne de la théorie de la guerre juste, n'est pas strictement identique au problème des droits qui nous occupe ici, car tous les dilemmes moraux ne sont pas formulés en termes de droits - historiquement, ils ne l'ont d'ailleurs été qu'à partir du XVII siècle - , et cette formulation, partagée à la fois par la philosophie (droits moraux) et par le droit (droits juridiques), force à l'interdisciplinarité.

Les droits humains en situation de conflits armés ne sont plus les droits abstraits, hypothétiques et souhaités dans lesquels il fallait «croire» pour les faire exister - dans l'esprit de certains en tout cas. Ils existent désormais en tant qu'obligations juridiques internationales inscrites dans de nombreux textes que quasiment tous les États sur la planète ont ratifiés, et qui correspondent à deux corpus bien définis du droit international: le droit international humanitaire (DIH) et le droit international des droits de l'homme (DIDH), dont aucune analyse philosophique sérieuse ne devrait faire l'économie, si elle veut parler du monde réel. Pour cette raison, on ne s'étonnera pas de la couleur juridique des pages suivantes: toute réflexion d'éthique des relations internationales, dont celle-ci fait partie, est à l'intersection d'au moins trois disciplines (philosophie, science politique, droit) et doit donc pratiquer l'interdisciplinarité pour être non seulement crédible mais même intelligible ${ }^{3}$.

1. I. Kant, Doctrine du droit, II, $\mathbb{S}_{53}$, in Métaphysique des moeurs II, Paris, GF Flammarion, I994, p. I67-I68.

2. B. Orend, «La justice après la guerre. Pour une nouvelle convention de Genève appliquée au jus post bellum», Raisons politiques, 45, 20I2, p. I63-I 86.

3. Pour une défense de l'interdisciplinarité, voir J.-B. Jeangène Vilmer et R. Chung, Éthique des relations internationales. Problématiques contemporaines, Paris, PUF, p. 4-7. 
Les relations entre droits humains et conflits armés sont résumées dans cet article en quatre parties: d'un point de vue historique, d'abord, où l'on montre leur réciprocité (le rôle de la guerre dans l'évolution des droits humains, et le rôle des droits humains dans l'évolution de la guerre). En reprenant la trilogie de l'éthique de la guerre, ensuite: la guerre au nom des droits humains (jus ad bellum), les droits humains dans la guerre (jus in bello) et, en guise de conclusion, les droits humains après la guerre (jus post bellum).

\section{Histoire: la guerre a fait les droits, et les droits ont fait la guerre}

«La guerre a fait l'État, et l'État a fait la guerre ${ }^{4}$ ». Cette formule fameuse de Charles Tilly résume bien la théorie belliciste de la formation de l'État, qui soutient que la guerre, en puissance (sa préparation) ou en acte (sa conduite), est une activité génératrice et organisatrice de l'État. Bien entendu elle n'est pas la seule, la cause des États est multifactorielle, et parfois la guerre ne fait pas d'État, et ce non-État continue néanmoins à faire la guerre ${ }^{5}$ (aujourd'hui, la plupart de ceux qui font la guerre sont des acteurs non étatiques). La formule ne doit donc pas s'entendre trop littéralement.

$\mathrm{Si}$, tout en gardant à l'esprit ces limites, l'on remplace l'État par les droits humains - ce qui n'est pas saugrenu puisque les seconds sont la production du premier (ce sont les États qui ont construit, peu à peu, un régime de DIDH; l'ONU, la Déclaration universelle des droits de l'homme (DUDH), la Cour pénale internationale (CPI), la Cour européenne des droits de l'homme (CEDH), sont leurs œuvres) —, la formule de Tilly fonctionne encore: la guerre a fait les droits humains, et les droits humains ont fait la guerre. Cette seconde partie s'entend toutefois dans un sens différent: les droits humains ne sont certes pas à l'origine de la guerre, qui existait bien avant eux, mais ils ont donné une raison supplémentaire de la faire.

\section{Le rôle de la guerre dans l'évolution des droits humains}

Il est consensuel d'affirmer que la DUDH est née des cendres de la Seconde Guerre mondiale 6 . Elle est «née du besoin de réaffirmer ces droits après la violation qu'ils avaient subie pendant la guerre ", explique par exemple la représentante de l'Inde à l'époque ${ }^{7}$. La DUDH étant « le point de départ réel,

4. C. Tilly, The Formation of National States in Western Europe, Princeton, Princeton University Press, I975, p. 42.

5. R. Cohen, "Warfare and State Formation: Wars Make States and States Make Wars ", in B. R. Ferguson (dir.), Warfare, Culture, and Environment, Orlando, Academic Press, I984, p. 329-358.

6. Pour toute cette section, voir X. Aurey, «Déclaration universelle des droits de l'homme et conflits armés: de la fragmentation à la complexité ", Cahiers de la recherche sur les droits fondamentaux (CRDF), 7, p. 49-62.

7. UN Doc. A/PV.r 82 ( Io décembre I948). 
le fondement » du DIDH ${ }^{8}$, c'est de tout le mouvement des droits humains du $\mathrm{Xx}^{\mathrm{e}}$ siècle dont la guerre aurait accouché, en réaction à ses atrocités9

Le préambule de la Charte de l'ONU confirme cet héritage - des deux guerres mondiales, pas seulement de la seconde — , en proclamant non seulement sa «foi dans les droits fondamentaux de l'homme» mais aussi son objectif de "préserver les générations futures du fléau de la guerre qui deux fois en l'espace d'une vie humaine a infligé à l'humanité d'indicibles souffrances".

Le mouvement des droits humains est né de la guerre, mais il s'est aussitôt sevré. En dépit des efforts de René Cassin "pour que l'on conservât cette trace de la guerre dans le texte» de la DUDH, il n'en reste pas grandchose. Trois ans après la Charte de l'ONU mettant la guerre hors-la-loi (le droit de la Charte est un jus contra bellum $)^{10}$, la Déclaration ne pouvait pas lui faire la part belle: elle était plutôt conçue comme un texte pour la paix. Réciproquement, les Conventions de Genève qui, l'année suivante (I949), contribuent grandement à l'édification du DIH, c'est-à-dire du droit de la guerre (au sens de droit dans la guerre, jus in bello), ne sont pas - ou peu ${ }^{11}$ - influencées par les droits humains. Autrement dit, dès les premières années de l'après-Seconde Guerre mondiale, la division du travail est nette: même s'ils partagent une origine commune - le respect, en tout temps, de la personne humaine - , le DIH régule le temps de la guerre, et le DIDH celui de la paix.

$\mathrm{Si}$, donc, le mouvement des droits humains est né de la guerre, il s'en arrache très vite. Cette méfiance a une raison plus profonde: historiquement, les droits humains relèvent de la sphère interne des États. De l'habeas corpus à la Déclaration des droits de l'homme et du citoyen française en passant par les déclarations d'indépendance américaines, ils "ont trait à l'organisation du pouvoir étatique face à l'individu. Ils sont le produit des théories du siècle des Lumières sur l'État et ont tout naturellement trouvé leur expression dans le droit constitutionnel interne ${ }^{12}{ }^{»}$. Leur cadre conceptuel est celui de la souveraineté.

Or, contrairement à une idée reçue, cette souveraineté qui caractérise l'ordre westphalien depuis I 648 n'a jamais été absolue: elle est plutôt une

8. G. Cohen-Jonathan et J.-F. Flauss (dir.), Droit international. Droits de l'homme et juridictions internationales, Bruxelles, Bruylant, 2004 , p. I2.

9. T. Meron, "International Law in the Age of Human Rights", Recueil des cours de l'Académie de droit international, 301, 2003, p. 29.

10. O. Corten, Le droit contre la guerre. L'interdiction du recours à la force en droit international contemporain, Paris, Pedone, 2008.

11. R. Provost y voit une influence dans les dispositions interdisant les discriminations et celles sur la torture et les traitements cruels, inhumains ou dégradants (International Human Rights and Humanitarian Law, Cambridge, Cambridge University Press, 2002, p. 6).

12. R. Kolb, "Relations entre le droit international humanitaire et les Droits de l'homme ", Revue internationale de la Croix-Rouge, 83 I, I998, p. 437. 
"construction rhétorique ${ }^{13}$ » ou, comme le montre Krasner, une «hypocrisie organisée $^{14}$ »- d'ailleurs déjà remise en cause par les juristes du XIX ${ }^{e}$ siècle qui critiquaient la "théorie de la souveraineté absolue [...] comme un dogme "archaïque", "inutile", "impraticable”, "décevant" ou même "dangereux", comme une "fiction néfaste" qui ne correspond plus aux faits de la vie internationale et est en réalité incompatible avec l'existence d'une société d'États gouvernée par un système de droit international ${ }^{15}$ », et qui préféraient parler de souveraineté conditionnelle - c'est-à-dire conditionnée au respect des droits humains.

La remise en cause du dogme de la souveraineté absolue de l'État n'est donc pas nouvelle, mais elle va encore se renforcer avec l'Holocauste: c'est derrière le paravent de la souveraineté que se cachait déjà Goebbels en I933 lorsqu'il répondait à la Société des Nations aux inquiétudes sur le sort des juifs allemands en disant que "charbonnier est maître chez soi ». C'est à cette crise de la souveraineté qu'à l'issue de la Seconde Guerre mondiale la Charte, les tribunaux de Nuremberg et Tokyo puis la DUDH répondent: ils internationalisent les droits humains, c'est-à-dire reconnaissent et même institutionnalisent le fait qu'ils ne relèvent pas uniquement de la sphère interne de l'État.

Réciproquement, les droits humains individualisent le droit international dont, traditionnellement, seuls les États sont sujets, et qui doit désormais protéger aussi des êtres humains - du génocide, des crimes contre l'humanité, crimes de guerre, nettoyage ethnique et plus généralement des violations des droits humains. Ce n'est pas seulement une évolution du droit international, observe à juste titre Xavier Aurey, mais une véritable révolution au sens kuhnien du terme, c'est-à-dire un changement de paradigme ${ }^{16}$ : la société internationale n'est plus (qu') une société des États, mais tend vers une société humaine en principe universelle.

\section{Le rôle des droits humains dans l'évolution de la guerre}

La guerre a donc joué un rôle dans l'évolution des droits humains, plus précisément dans l'élaboration d'un DIDH dans l'après-Seconde Guerre mondiale. Inversement, les droits humains ont aussi joué un rôle dans l'évolution des conflits armés, et depuis plus longtemps, en constituant l'une des causes d'usage de la force. C'est la naissance à la fin du XIX ${ }^{e}$ siècle de ce qu'on appe-

13. P. Cunliffe, "A Dangerous Duty: Power, Paternalism and the Global 'Duty of Care' ", in P. Cunliffe (ed.), Critical Perspectives on the Responsibility to Protect: Interrogating Theory and Practice, Londres, Routledge, 20I I, p. 60.

14. S. Krasner, Sovereignty: Organized Hypocrisy, Princeton, Princeton University Press, I999.

15. J. Garner, "Des limitations à la souveraineté nationale dans les relations extérieures", Revue de droit international et de législation comparée, 6, I925, p. 37.

16. X. Aurey, "Déclaration universelle des droits de l'homme et conflits armés ", op. cit., p. 52 . 
lait à l'époque l' "intervention d'humanité ». Les premiers théoriciens d'un «droit d'intervention» sont majoritairement des juristes inspirés par la Question d'Orient, c'est-à-dire les interventions des puissances européennes dans l'Empire ottoman entre I 827 et I908 pour protéger des minorités, notamment chrétiennes.

Cet essor de l'interventionnisme peut s'expliquer par plusieurs facteurs, dont le développement des droits humains comme l'un des attributs des "peuples civilisés ", l'impact de la sensibilité religieuse (sur le mouvement pour l'abolition de l'esclavage notamment) et le développement des moyens de communication et de projection des forces à l'international ${ }^{17}$.

Les juristes interventionnistes invoquent "les droits de l'humanité18", les «droits de l'homme» et les «droits humains": «Chaque fois que les droits bumains d'un peuple seraient méconnus par ses gouvernants, explique Rougier, un ou plusieurs États pourraient intervenir au nom de la Société des nations ${ }^{19}$.» Amédée Bonde est contre tout gouvernement qui «viole les droits de l'humanité par des excès d'injustice et de cruauté envers certaines catégories de sujets au mépris des lois de la civilisation ${ }^{20}$ ». «La proclamation des droits de l'homme doit donc obliger toutes les nations, et le respect de ces droits doit être assuré contre les écarts éventuels de tous les États, sans exception aucune ${ }^{21}$ ", renchérit Mandelstam, qui défend «un Pacte des peuples civilisés proscrivant la violation des droits de l'homme et créant la probabilité d'une intervention d'humanité ». Dix-huit ans avant la DIDH, il appelait déjà à «la conclusion d'une convention mondiale sur la protection des droits de l'homme $22 »$.

Cette question de l'intervention d'humanité — ou humanitaire, comme on dit aujourd'hui - est l'interaction la plus évidente entre droits humains et conflits armés: une relation de causalité (la protection des droits humains est susceptible de causer un conflit armé, ou en tout cas de contribuer à son déclenchement car les motivations sont toujours mixtes et les causes multifactorielles). Elle est aussi la première de la trilogie de la guerre juste, au niveau du jus ad bellum.

17. M. Marrus, «International Bystanders to the Holocaust and Humanitarian Intervention", in R. A. Wilson et R. D. Brown (eds.), Humanitarianism and Suffering: The Mobilization of Empathy, Cambridge, Cambridge University Press, 2009, p. I62.

$18 \mathrm{M}$. Kebedgy, De la tutelle des femmes pubères et De l'intervention: théorie générale et étude spéciale de la Question d'Orient, Thèse pour le doctorat à la Faculté de droit de Paris, Paris, A. Giard, I890, p. 46.

19 A. Rougier, "La théorie de l'intervention d'humanité », Revue générale de droit international public, I7, I9I0, p. 472. L'expression "Société des nations" est à prendre au sens figuré, car la SDN n'existe pas encore.

20 A. Bonde, Traité élémentaire de droit international public, Paris, Dalloz, I926, p. 245 .

21 A. Mandelstam, "La généralisation de la protection internationale des droits de l'homme», Revue de droit international et de législation comparée, I I, I930, p. 700-70I.

22 Ibid., p. 7I 2. 


\section{La guerre au nom des droits humains (jus ad bellum)}

Pour comprendre ce qu'Antonio Cassese a dit des droits humains - que leur arrivée sur la scène internationale est « un événement remarquable car ils sont une théorie subversive destinée à favoriser les tensions et les conflits entre les États ${ }^{23}$ » - , il faut revenir à la tension originelle, dans la Charte, entre la souveraineté, c'est-à-dire la non-intervention (art. 2 [4]), et la promotion des droits humains (art. I [3]): car il y a des situations dans lesquelles protéger les droits humains implique de violer la souveraineté, c'est-à-dire intervenir militairement en territoire étranger sans le consentement de l'État cible. C'est typiquement le cas lorsque les exactions qu'il s'agit de faire cesser sont le fait du gouvernement, soit directement (il en est l'auteur), soit indirectement (il les laisse faire). Et c'est dans ce contexte que se pose la question de l'intervention humanitaire ou, pour éviter cette expression discutable, de l'intervention militaire justifiée par des raisons humanitaires.

\section{L'intervention humanitaire}

C'est une question difficile puisque, la Charte ayant mis la guerre hors-laloi, l'intervention est par défaut illégale sauf si elle se fait avec le consentement de l'État, est autorisée par le Conseil de sécurité sous le chapitre VII (ou relève de la légitime défense, mais puisqu'en l'occurrence les droits humains qu'il s'agit de protéger sont ceux de populations étrangères, l'intervention humanitaire n'appartient pas a priori à cette catégorie). Lorsque c'est le cas, comme en Libye (20I I), la justification est relativement simple, même si elle sera toujours discutable puisque certains contestent que la menace que Kadhafi faisait peser sur les populations était suffisamment grave pour justifier une intervention ou, a posteriori (ce qui est toujours plus facile), l'effet positif de celle-ci. Lorsque ce n'est pas le cas, en revanche, c'est-à-dire lorsque le Conseil de sécurité n'autorise pas l'intervention, comme au Kosovo (I999) ou comme l'envisageaient certains pays début septembre $20 \mathrm{I} 3$ au sujet de la Syrie, la justification est plus compliquée ${ }^{24}$.

Elle repose alors sur un argument moral: la souveraineté n'est pas un dû, elle se mérite, elle implique des obligations, dont celle de respecter les droits de l'homme sur son territoire. L'État qui viole ces obligations perd du même coup la protection de la souveraineté, et ipso facto le droit de ne pas subir d'intervention extérieure, en même temps que des États tiers bien

23 A. Cassese, International Law, 2nd ed., Oxford, Oxford University Press, 2005, p. 375 .

24. Les interventions occidentales en Syrie en 2015 se font également sans le consentement d'Assad, ni l'autorisation du Conseil de sécurité, mais sont justifiées par la légitime défense (collective et individuelle), et non par la responsabilité de protéger les populations syriennes. 
intentionnés gagnent le droit d'intervenir ${ }^{25}$. C'était déjà le raisonnement des juristes interventionnistes $\mathrm{du} \mathrm{XIX}^{\mathrm{e}}$ siècle qui défendaient la souveraineté conditionnelle: le droit ne protège que les États «réellement dignes de ce nom ${ }^{26}$ »; «la souveraineté ne doit être respectée que lorsqu'elle est respectable; or elle ne l'est pas quand elle viole les devoirs internationaux ${ }^{27}$ ", etc.

Plus récemment, Annan affirmait au tournant du millénaire qu' «en cas de violations massives des droits de l'homme universellement acceptées, nous avons la responsabilité d'agir ${ }^{28}$ ».

La souveraineté ne peut plus être un bouclier pour protéger ceux qui violent les droits en toute impunité. Quand un État échoue à protéger sa population, la communauté internationale doit intervenir et ceux qui sont menacés de génocide, nettoyage ethnique, crimes de guerre ou crimes contre l'humanité ont le droit de compter sur cette aide ${ }^{29}$.

Les interventionnistes (favorables à une intervention unilatérale, c'està-dire sans autorisation du Conseil de sécurité, dans certaines situations et à certaines conditions) partageant cette conviction se divisent alors en deux camps $^{30}$. D'un côté, les partisans de l'approche par la doctrine qui, comme les juristes interventionnistes un siècle plus tôt (Arntz, Rolin-Jaequemyns, Rougier, Fiore, Wheaton, Woolsey, Borchard et Lingelbach), défendent un droit d'intervention ${ }^{31}$, c'est-à-dire une réforme du droit. Rougier, par exemple,

25. C. Beitz, "Bounded Morality: Justice and the State in World Politics", International Organization, 33:3, 1979, p. 405-424 et «Human Rights as a Common Concern ", American Political Science Review, 95: 2, 200I, p. 269-282; D. Luban, "Just War and Human Rights", Philosophy and Public Affairs, 9:2, I980, p. I60-I8I; R. Keohane, "Political Authority After Intervention: Gradations in Sovereignty ", in J. L. Holzgrefe et R. O. Keohane (eds.), Humanitarian Intervention: Ethical, Legal, and Political Dilemmas, Cambridge, Cambridge University Press, 2003 , p. 275-298; F. Tesón, "The Liberal Case for Humanitarian Intervention », in J. L. Holzgrefe et Robert O. Keohane (eds.), Humanitarian Intervention: Ethical, Legal, and Political Dilemmas, Cambridge, Cambridge University Press, 2003, p. 93-I29; H. Shue, "Limiting Sovereignty", in J. M. Welsh (ed.), Humanitarian Intervention and International Relations, Oxford, Oxford University Press, 2004, p. I I et C Chwaszcza, Moral Responsibility and Global Justice: A Human Rights Approach, Baden-Baden, Nomos, 2007, p. I2I-I 22.

26. G. Rolin-Jaequemyns, «Le droit international et la phase actuelle de la question d'Orient ", Revue de droit international et de législation comparée, 8, I876, p. 369.

27. P. Fauchille, Traité de droit international public, $8^{\mathrm{e}}$ éd., Paris, Rousseau \& Cie, I922, p. 565 .

28. Rapport du Secrétaire général sur l'activité de l'Organisation, $2000,55^{\mathrm{e}}$ session, Suppl. ${ }^{\circ}$ I, UN Doc. A/55/I, $\$ 37$, p. 5 .

29. K. Annan, "Foreword", Global Responsibility to Protect, 3, 20 I I, p. 382.

30. N. Wheeler, Saving Strangers: Humanitarian Intervention in International Society, Oxford, Oxford University Press, 2000, p. 33-5I; S. Chesterman, Just War or Just Peace? Humanitarian Intervention and International Law, Oxford, Oxford University Press, 200I, p. 226-232 et R. Thakur, The United Nations, Peace and Security: From Collective Security to the Responsibility to Protect, Cambridge, Cambridge University Press, 2006, p. 260.

31. UK Foreign and Commonwealth Office, Is Intervention Ever Justified?, Planning Staff, Foreign Policy Document No I48, London, I984, II, 22, p. 8 et C. Greenwood, «Legal 
opposait à la "philosophie humanitaire et imprécise du XVIII ${ }^{\mathrm{e}}$ siècle» le progrès et la rigueur du droit ${ }^{32}$.

De l'autre, les partisans de l'approche par l'exception, qui pensent que l'intervention est un problème moins juridique que politique et moral, qui ne contestent pas l'illégalité de l'intervention, mais pensent que, sans créer de précédent, celle-ci doit être permise à titre exceptionnel ${ }^{33}$. On parle alors d'une intervention «illégale mais légitime» ou "illégale mais morale ${ }^{34}$. Comme l'euthanasie, expliquait Brownlie dans une analogie célèbre en I973, l'intervention humanitaire est illégale mais excusable ${ }^{35}$. Cette seconde approche, que j'ai défendue ailleurs ${ }^{36}$, consiste à faire primer, dans certaines situations exceptionnelles, la légitimité sur la légalité, et permet d' "envisager l'hypothèse de violations "éthiques" du droit plutôt que de vouloir réformer le droit à tout prix ${ }^{37}$ ».

\section{La dimension identitaire}

Les États n'invoquent pas tous autant les droits humains pour faire la guerre, et ces différences s'expliquent par des raisons identitaires, c'est-à-dire par la manière dont chacun se représente sa relation avec les droits humains. Parmi les nombreux États dans le monde qui contestent l'argument «libéral» en faveur de l'intervention humanitaire, certains le font sur la base d'un relativisme culturel: parce qu'ils contestent par ailleurs l'universalité des droits de

Justification for the Resort to Force ", in P. Mileham (ed.), War and Morality. Proceedings of a RUSI Conference: 'Morality in Asymetric War and Intervention Operations'held on 19-20 September 2002, Whitehall Paper 6I, London, The Royal United Services Institute for Defence and Security Studies, 2004, p. 40.

32. A. Rougier, "La théorie de l'intervention d'humanité", op. cit., p. 472.

33. O. Schachter, International Law in Theory and Practice, Dordrecht, Martinus Nijhoff, I99I, p. I26; L. Henkin, "Kosovo and the Law of "Humanitarian Intervention" ", American Journal of International Law, 93:4, I999, p. 826; et T. Franck, Recourse to Force. State Action Against Threats and Armed Attacks, Cambridge, Cambridge University Press, 2002, p. I 8 I.

34. Au sujet du Kosovo, approche par exemple défendue par l'ensemble des articles du numéro 93 du American Journal of International Law de I999 à une exception près (Chinkin); B. Simma, "NATO, the UN and the Use of Force: Legal Aspects ", European Journal of International Law, IO: I, I999, p. I-22; The Independent International Commission on Kosovo, The Kosovo Report: Conflict, International Response, Lessons Learned, Oxford, Oxford University Press, 2000, p. I 86; M. Koskenniemi, " "The Lady Doth Protest Too Much": Kosovo, and the Turn to Ethics in International Law", Modern Law Review, 65: 2, 2002, p. I 59-I75, etc.

35. I. Brownlie, "Thoughts on Kind-Hearted Gunmen", in R. B. Lillich (ed.), Humanitarian Intervention and the United Nations, Charlottesville, University of Virginia Press, I973, p. 146.

36. J.-B. Jeangène Vilmer, La Guerre au nom de l'humanité. Tuer ou laisser mourir, Paris, PUF, 20I 2.

37. F. Mégret, "L'éthique de non-intervention du droit international», in J.-F. Rioux (dir.), L'intervention armée peut-elle être juste? Aspects moraux et éthiques des petites guerres contre le terrorisme et les génocides, Montréal, Fides, 2007, p. I70. 
l'homme, une «invention occidentale », et érigent des modèles de remplacement. L'argument relativiste avait jusqu'alors trois sources: une autocritique de l'Occident lui-même, l'islam et l'Extrême-Orient ${ }^{38}$.

On connaissait la revendication de "valeurs asiatiques ", dont il existe deux versions: une forte selon laquelle le concept même de droits humains est occidental et donc inapplicable à l'Asie; une faible qui considère que le concept est en lui-même universel mais qu'il est approprié d'en avoir différentes conceptions pour différentes cultures. En l'occurrence, la Chine utilise la scission classique entre droits "libéraux» (civils et politiques) et droits "socialistes» (économiques, sociaux et culturels) comme un prétexte pour justifier sa conception "différente» des droits de l'homme ${ }^{39}$.

Li Peng, Premier ministre de la République populaire de Chine entre I987 et I998, lié notamment au massacre de la place Tiananmen et accusé de génocide au Tibet par la Cour suprême d'Espagne en 2006, explique que "les droits de l'homme relèvent de la souveraineté nationale [...]. La Chine en fait grand cas, mais elle s'oppose à l'ingérence dans les affaires intérieures des autres pays en utilisant les droits de l'homme comme excuse $^{40}$ ». Durant l'affaire du Kosovo, l'ambassadeur chinois au Conseil de sécurité dit également que "l'approche qui fait primer les droits de l'homme sur la souveraineté » les utilise comme prétexte pour viser l'hégémonie ${ }^{41}$.

Aujourd'hui, c'est la Russie, plus que la Chine, qui tente de devenir un pôle d'attraction différent - avec une dimension non seulement territoriale (Union eurasiatique versus UE) mais aussi normative (conservatisme, "rappeler l'Europe à ses propres valeurs gréco-romaines $)^{42}$. Cette phase agressive pourrait toutefois ne pas survivre à Poutine.

Parmi les États qui, au contraire, sont les plus attachés à l'idée de l'intervention humanitaire, deux en particulier revendiquent une relation particulière avec les droits humains, et - car ce n'est pas suffisant - ont une prétention à l'universalité, c'est-à-dire s'attribuent un rôle messianique dans les affaires du monde: la France et les États-Unis. Si ces deux États ont développé des doctrines interventionnistes - le «droit ou devoir d'ingérence» en France, la «humanitarian intervention» du wilsonisme

38. M. Ignatieff, «The Attack on Human Rights », Foreign Affairs, 80: 6, 200I, p. I02.

39. J. Bauer et D. Bell (eds.), The East Asian Challenges for Human Rights, Cambridge, Cambridge University Press, I999 et J. Donnelly, International Human Rights, 3 rd ed., New York, Westview, 2007, p. 162-166.

40. L. Peng, "Chinese Views on a New World Order (Chinese Premier Li Peng's speech at the Summit Meeting of the UN Security Council on January 3 I, I992) ", Beijing Review, 35 : 7, I7-23 février I992, p. I4.

41. Résolution I 244, SCOR, 40IIth Mtg, Io juin I999, p. 9.

42. Alexandre Douguine, cité dans M. Eltchaninoff, "Dans la tête de Vladimir Poutine ", Philosophie magazine, 80 , juin 2014. 
américain $^{43}$ - chacun revendiquant d'ailleurs la paternité du concept, c'est en partie pour une question d'image de soi et d'identitét4.

La France, parce qu'elle se persuade d'être la "patrie des droits de l'homme» - ce qui est faux. Robert Badinter parle de "cécité historique» et aime rappeler que la France n'est pas la patrie des droits de l'homme mais de la déclaration des droits de l'homme, ce qui n'est pas la même chose. Cette déclaration s'appuyant elle-même sur des textes antérieurs américains et anglais, la France n'est pas davantage la patrie des droits de l'homme que les États-Unis et l'Angleterre.

Cette hypertrophie déclaratoire peut d'ailleurs être contreproductive dans au moins deux cas de figure. D'une part, lorsqu'elle est exclusive des intérêts: prétendre que nous agissons parfois de manière totalement désintéressée, comme nous l'avons fait pour justifier l'intervention en Libye — alors qu'Obama et Cameron reconnaissaient la présence d'intérêts nationaux aux côtés de la raison humanitaire - attise la suspicion et renforce le cynisme de l'opinion publique qui n'est pas dupe. Il est important d'assumer la présence de l'intérêt et de reconnaître qu'il n'est pas incompatible avec la morale.

D'autre part, lorsqu'elle n'est pas suivie d'effet: parler de «ligne rouge » en Syrie pour finalement ne pas frapper lorsqu'elle est franchie, par exemple, nuit à l'image et à la crédibilité de la France. Avoir un discours dur sur l'Ukraine si nous ne sommes pas prêts à aller loin, aussi. Cela ne signifie pas que le purement déclaratoire est inutile — sur les États sensibles à leur image morale, il peut être efficace - mais que sur ceux qui sont soit trop forts (Russie) soit déjà parias (Syrie), il est non seulement inefficace mais même potentiellement nuisible, et doit donc être manipulé avec prudence.

Quant aux États-Unis, leur interventionnisme est ancré dans des valeurs qu'ils estiment consubstantielles à leur identité - droits humains, liberté et démocratie, qui génèrent la tentation libérale d' 'imposer Locke partout ${ }^{45}$ »-, et aussi dans une volonté de conforter leur leadership, qui implique selon eux une responsabilité d'agir. Ce lien entre puissance et responsabilité est typique du discours américain ${ }^{46}$.

Dans tous les cas - celui de la France comme celui des États-Unis -, cette relation identitaire aux droits humains qui incite à l'interventionnisme passe de moins en moins dans un contexte où leur verticalité, c'est-à-dire la suprématie occidentale des droits humains, est de plus en plus contestée.

43. Sur la renaissance du wilsonisme américain, voir A. Coll, "Kosovo and the Moral Burdens of Power", in A. J. Bacevich et E. A. Cohen (eds.), War Over Kosovo: Politics and Strategy in a Global Age, New York, Columbia University Press, 200I, p. I25-I 26.

44. N. Crawford, Argument and Change in World Politics: Ethics, Decolonization, and Humanitarian Intervention, Cambridge, Cambridge University Press, 2002, p. I I 4.

45. L. Hartz, The Liberal Tradition in America, New York, Harvest Books, I955, p. I3.

46. C.-H. Chang, Ethical Foreign Policy? US Humanitarian Interventions, Farnham, Ashgate, 20I I, p. I 55-I 57 . 


\section{Quels droits humains?}

Parmi les nombreuses questions que pose l'intervention humanitaire, celle de la nature du dommage (pourquoi intervenir exactement?), constitutive de la cause juste dans les termes de la théorie traditionnelle de la guerre juste, suscite généralement deux types de réponses: celles formulées en termes d'exactions (génocide, crimes contre l'humanité, nettoyage ethnique, crimes de guerre à grande échelle, etc.) et celles formulées en termes de droits.

Dire que l'intervention humanitaire consiste à intervenir militairement en cas de "violations des droits humains", comme le font la plupart des auteurs, manque de précision et permet d'abuser du concept: il y a des dizaines de droits humains et, si l'on ne dit pas lesquels sont concernés, alors peut se prétendre «humanitaire » quasiment pour n'importe quelle intervention. On observe que les opérations militaires ne sont pas déclenchées pour protéger la liberté d'expression, le droit de vote ou le droit de grève. Quels droits alors?

Il y a un siècle, Rougier parlait de la «violation manifeste des droits essentiels de l'humanité ${ }^{47}$ ", qu'il explicitait en droit à la vie, droit à la liberté et droit à la légalité. Le premier est le plus consensuel, nous y reviendrons. Les deux autres sont plus originaux. Rougier restreint le droit à la liberté susceptible de déclencher une intervention à la liberté physique seulement, qui prohibe l'esclavage par exemple ${ }^{48}$. La répression de l'esclavage était considérée par plusieurs auteurs de cette époque comme une cause juste d'intervention ${ }^{49}$. Plus discutée en revanche était la question de savoir s'il fallait étendre cette protection à la liberté religieuse ${ }^{50}$.

D'autres ont des critères bien plus larges: droit à la propriétées1, situation d'anarchie ou d'État "défaillant", guerre civile ${ }^{52}$, rébellion ${ }^{53}$, etc. Le seuil des causes justes légitimant l'intervention est globalement assez bas:

47. A. Rougier, Les guerres civiles et le droit des gens, Thèse pour le doctorat de sciences politiques, Paris, Librairie de la société du recueil général des lois et des arrêts, I902, p. 345.

48. A. Rougier, "La théorie de l'intervention d'humanité", op. cit., p. 5 I8.

49. M. Kebedgy, op. cit., p. 85 et 86; P. Fauchille, op. cit., p. 57I ; O. Löwenheim, " "Do Ourselves Credit and Render a Lasting Service to Mankind": British Moral Prestige, Humanitarian Intervention, and the Barbary Pirates ", International Studies Quarterly, 47: I, 2003, p. 23.

50. P. Fauchille, op. cit., p. 57I ; E. Stowell, «Intervention d'humanité », in «La théorie et la pratique de l'intervention", Recueil des cours de l'Académie de droit international, 40, I932, p. I4 I et L. Le Fur, "L'intervention pour cause d'humanité", in Vitoria et Suarez: contribution des théologiens au droit international moderne, Paris, Pedone, I939, p. 230.

51. P. Fauchille, op. cit., p. 57I.

52. A. Heffter, Le droit international public de l'Europe, Berlin, E. H. Schroeder, I 857 , p. I0 5 et H. Halleck, International Law, or Rules Regulating the Intercourse of States in Peace and War, San Francisco, H. H. Bancroft \& co, I961, p. 340.

53. S. Amos, Lectures on International Law, London, Stevens \& Sons, I874, p. 40 et W. Manning, Commentaries on the Law of Nations, new ed., London, I875, p. 97. 
selon ces critères, il serait aujourd'hui justifié d'intervenir contre n'importe quelle dictature, même tranquille, et sans qu'il y ait une urgence humanitaire à proprement parler.

La pratique contemporaine des États montre au contraire que l'intervention humanitaire a lieu en cas de violations d'un seul droit: le droit à la vie. Et encore, seulement des violations les plus graves, car il ne s'agit pas d'intervenir pour un meurtre isolé. À partir de combien de meurtres, et de quels types de meurtres, peut-on parler d'un massacre justifiant une intervention extérieure? Dans quelle mesure, même, les nombres doivent-ils compter? C'est un débat classique en philosophie morale ${ }^{54}$, qui trouve d'ailleurs une application dans le cas de la qualification de génocide, puisqu'elle ne dépend pas tant du nombre des victimes que de l'intention des meurtriers $(\text { mens rea })^{55}$.

Le noyau dur, relativement consensuel, des causes justes est constitué des quatre crimes identifiés dans la version onusienne de la responsabilité de protéger $\left(\mathrm{R}_{2} \mathrm{P}\right)^{56}$, c'est-à-dire les articles I38-I39 du document final du Sommet mondial de l'ONU en 2005 . Ils affirment notamment que "c'est à chaque État qu'il incombe de protéger ses populations du génocide, des crimes de guerre, du nettoyage ethnique et des crimes contre l'humanité»

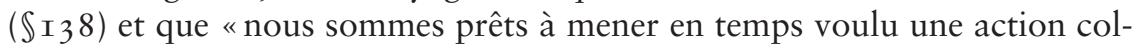
lective résolue, par l'entremise du Conseil de sécurité, [...] lorsque ces moyens pacifiques se révèlent inadéquats et que les autorités nationales n'assurent manifestement pas la protection de leurs populations» ( $\mathbb{S}$ I39).

Que ce texte fasse par ailleurs référence au chapitre VII est révélateur, car il implique que ce n'est pas la violation des droits humains par un État qui en soi active l'usage de la force, mais le fait qu'elle menace la paix et la sécuritée $^{57}$. Cela réduit donc les chances que le texte soit appliqué puisqu'il ne faut pas seulement qu'il y ait une catastrophe humanitaire, il faut encore prouver qu'elle constitue une menace à la paix et à la sécurité.

Pour refléter cette interprétation très restrictive des droits humains dont la violation est susceptible de déclencher une intervention militaire, il faut donc définir l'intervention humanitaire comme celle visant à prévenir ou arrêter des violations graves et massives des droits les plus fondamentaux

54. J. Taurek, "Should the Numbers Count?», Philosophy and Public Affairs, 6, I977, p. 293-3I6.

55. J.-B. Jeangène Vilmer, "La responsabilité de protéger et le débat sur la qualification de génocide au Darfour", La responsabilité de protéger, Actes du $4 \mathrm{I}^{\mathrm{e}}$ colloque annuel de la Société française pour le droit International (SFDI), Paris, Pedone, 2008, p. 233-24I.

56. Qui diffère sensiblement de la version initiale de la CIISE, La responsabilité de protéger, Ottawa, Centre de recherches pour le développement international, 200I. Voir J.-B. Jeangène Vilmer, La responsabilité de protéger, "Que sais-je? ", PUF, 20 I 5.

57. J. Welsh, «The Responsibility to Protect: Securing the Individual in International Society ", in B. J. Goold et L. Lazarus (eds.), Security and Human Rights, Portland, Hart Publishing, 2007, p. 379. 
— ce qui pose un autre problème: celui de la hiérarchie de droits pourtant réputés indivisibles.

Formuler la nature du dommage en termes de droits ou en termes d'exactions - l'un revenant finalement à l'autre puisqu'il faut dire, pour ce qui est des exactions, quelles violations du droit à la vie sont concernées peut finalement s'avérer trop rigide: il n'existe pas une liste précise de droits, ou de crimes, en vertu desquels un État peut automatiquement intervenir dans un autre. L'intervention humanitaire n'étant pas un droit au sens positif du terme, elle n'a pas de cause objective, et dépend plutôt d'une appréciation au cas par cas et en fonction d'autres critères (autorité légitime, dernier recours, proportionnalité, chances raisonnables de succès).

Il y a en outre le risque d'élargir le cercle des causes justes au-delà du noyau dur, pour l'étendre notamment à la tyrannie ${ }^{58}$. En Irak en 2003, les Américains ont confondu et inclus l'intervention pro-démocratique dans l'intervention humanitaire - ces deux espèces étant pourtant distinguées depuis le $\mathrm{XIX}^{\mathrm{e}}$ siècle ${ }^{59}$. Une difficulté supplémentaire vient du fait que le changement de régime peut être un moyen ou une conséquence de l'intervention humanitaire (c'est ce qui s'est passé en Libye en 20II), mais pas une cause.

Une autre extension possible va vers la sécurité alimentaire: c'est le droit à la subsistance dont parle Shue ${ }^{60}$. Certains auteurs envisagent que la cause juste d'une intervention armée puisse intégrer le droit à une nourriture suffisante, évoquant l'hypothèse d'un autocrate empêchant délibérément son peuple de manger ${ }^{61}$. Ou encore la santé suite à une catastrophe naturelle: c'est Kouchner qui invoque le droit d'ingérence et la $\mathrm{R}_{2} \mathrm{P}$ en 2008 face au cyclone Nargis en Birmanie - mais l'on a vite compris que, sans le consentement des autorités locales, une intervention armée tuerait davantage de civils qu'elle n'en sauverait, et donc que le critère de l'effet positif ne serait pas satisfait.

Pour contourner ces difficultés, j’ai défendu ailleurs une formulation en termes ni de droits ni d'exactions, mais de dommages ${ }^{62}: c^{\prime} e s t$ la nature du dommage causé par le crime, plutôt que le crime lui-même, qui devrait jus-

58. M. Reisman, "Coercion and Self-Determination: Construing Charter Article 2 (4)", American Journal of International Law, 78:3, I984, p. 642, A. D'Amato, "The Invasion of Panama Was a Lawful Response to Tyranny ", American Journal of International Law, 84: 2, I990, p. 5 I9; F. Tesón, «Ending Tyranny in Iraq ", Ethics \& International Affairs, I9: 2, 2005, p. I-20.

59. G. Carnazza Amari, "Nouvel exposé du principe de non-intervention ", Revue de droit international et de législation comparée, 5, I873, respectivement p. 53 I, 534 et 535.

60. H. Shue, Basic Rights: Subsistance, Affluence, and U.S. Foreign Policy, Princeton, Princeton University Press, I980.

61. S. Miller, "Collective Responsibility and Armed Humanitarian Intervention", in T. Coady et M. O'Keefe (eds), Righteous Violence: The Ethics and Politics of Military Intervention, Melbourne, Melbourne University Press, 2005, p. 54.

62. J.-B. Jeangène Vilmer, "Pourquoi intervenir? Le critère de la cause juste dans la théorie de l'intervention humanitaire armée", Critique internationale, 54, 20I2, p. I45-I68. 
tifier ou non l'intervention. Ce dommage ne doit pas être quantifié a priori - fixer un seuil aurait l'effet pervers de permettre aux criminels se gardant de le dépasser de poursuivre tranquillement leur œuvre (c'est le mot bien connu d'un diplomate serbe à Javier Solana, alors secrétaire général de l'OTAN: "A village a day keeps NATO away ») — mais il doit être de "grande ampleur», «imminent ou en cours» et finalement guidé par le principe conséquentialiste suivant: la cause est juste lorsque le dommage fait plus de victimes que n'en ferait l'intervention ${ }^{63}$.

Cela signifie concrètement que seuls les cas extrêmes, où l'on extermine un million de personnes en cent jours, par exemple, comme au Rwanda, constituent des causes justes claires et assez peu discutables, puisque dans ces cas il semble difficile de concevoir qu'une intervention militaire visant à stopper les massacres puisse avoir l'effet pervers de les aggraver. Cette position est minimaliste, dans le sens de Ruwen Ogien, et du même coup antipaternaliste ${ }^{64}$ - ce qui est utile pour éviter la connotation paternaliste, c'est-à-dire néo-colonialiste, d'une notion comme la «responsabilité de protéger» qui implique une relation de protecteur à protégé.

\section{La sélectivité}

L'un des problèmes majeurs de l'intervention humanitaire est celui de la sélectivité ou de l'inconsistance, "deux poids, deux mesures» en français, «double standard» en anglais. Pourquoi la Libye et pas la Syrie? Pourquoi le Kosovo et pas la Tchétchénie ? La question est récurrente à chaque intervention, et elle est légitime puisque, d'un côté, on prétend intervenir au nom d'une règle universelle - la protection des droits humains — et, de l'autre, on ne l'applique pas universellement. Les droits des Tchétchènes, des Érythréens et des Darfouris valent-ils donc moins que ceux des Kosovars, des Timorais et des Libyens? Comment justifier cette asymétrie morale?

C'est en réalité un faux problème qui ne se pose qu'à ceux qui ont deux convictions : d'une part, que l'intervention est purement humanitaire, c'est-à-dire désintéressée; d'autre part, qu'il existe un devoir — pas seulement un droit - d'intervenir («devoir d'ingérence», R2P). Si les États n'interviennent que pour aider la population locale, alors il est effectivement incohérent d'intervenir ici et pas là si l'urgence humanitaire est similaire. Et si les États ont le devoir, donc l'obligation d'intervenir, on peut même les accuser de le faire en Libye mais pas en Syrie, au Kosovo mais pas en Tchétchénie.

Or ces deux convictions sont fausses. D'une part, la décision d'intervenir ne repose pas seulement sur le facteur humanitaire, mais sur un calcul

63. E. A. Heinze, Waging Humanitarian War: The Ethics, Law, and Politics of Humanitarian Intervention, Albany, State University of New York Press, 2009, p. 44-45.

64. R. Ogien, L'éthique aujourd'hui. Maximalistes et minimalistes, Paris, Gallimard, 2007 , p. I०8. 
coût/bénéfice qui inclut les risques encourus, à la fois pour les intervenants et les locaux, les intérêts nationaux en jeu et les conséquences politiques. Même dans l'hypothèse où l'urgence humanitaire serait similaire dans deux situations, les autres facteurs ne le sont pas forcément, et c'est pourquoi la décision finale n'est pas la même.

Les États n'interviennent que là où ils ont un intérêt à le faire. Rougier écrivait en I9IO: "Il se commet tous les jours dans quelque coin du monde mille barbaries qu'aucun État ne songe à faire cesser parce qu'aucun État n'a d'intérêt à les faire cesser ${ }^{65}$. » C'est toujours vrai. Toutes les interventions militaires justifiées par des raisons humanitaires ont été, sont et seront également motivées par des intérêts nationaux ${ }^{66}$. Délégitiment-ils l'intervention?

Certains le pensent, qui préfèrent renoncer à une intervention nécessaire parce qu'elle serait "polluée» par des intentions impures. Ceux-là vivent dans un monde idéal qui n'est pas le nôtre. Les motivations politiques sont inévitables et ne constituent pas en soi un problème. Elles ne le deviennent que lorsqu'elles sont contradictoires avec l'objectif humanitaire. Jusqu'à preuve du contraire, elles n'annulent pas comme par magie l'urgence, les besoins des victimes, les exactions commises.

D'autre part, le devoir d'ingérence est une mythologie française ${ }^{67}$ et la $\mathrm{R}_{2} \mathrm{P}$ ne doit pas être comprise au sens d'une obligation juridique: il y a éventuellement un droit d'intervenir lorsque le Conseil de sécurité l'autorise, ce qui est très différent. Il est assez paradoxal que les mêmes qui critiquent le rôle de "gendarme du monde" que se donneraient les États-Unis, la France, l'Angleterre ou l'Otan, leur reprochent d'intervenir ici mais pas là, comme s'il existait effectivement une loi qu'ils étaient chargés d'appliquer également partout.

L'intervention n'étant ni moralement désintéressée ni juridiquement obligatoire, on comprend mieux qu'elle soit par définition sélective et qu'intervenir en Libye pour des raisons qui sont propres à cette situation particulière n'implique aucunement devoir intervenir ailleurs, car la légitimité d'une intervention humanitaire ne se juge pas qu'à l'aune du seul critère de la cause juste, mais aussi en fonction de celui des conséquences probables. La raison d'être d'une intervention dite humanitaire est de sauver davantage qu'elle ne tue, et seules celles susceptibles de réaliser cet objectif doivent être envisagées.

65. A. Rougier, "La théorie de l'intervention d'humanité", op. cit., p. 525-526.

66. J.-B. Jeangène Vilmer, "Humanitarian Intervention and Disinterestedness ", Peace Review: A Journal of Social Justice, 19: 2, 2007, p. 207-216.

67 J.-B. Jeangène Vilmer, «De la mythologie française du droit d'ingérence à la responsabilité de protéger: une clarification terminologique ", Annuaire français des relations internationales, I3, 20I2, p. 8I-100. 
La tyrannie de la cohérence, qui voudrait qu'on intervienne soit partout soit nulle part, conduit en réalité à la politique du pire: faire la guerre à tout le monde, ou laisser mourir certaines victimes au motif qu'on ne peut pas les sauver toutes. Cette logique du tout ou rien s'applique bien mal à la réalité et n'est autre qu'un sophisme: ce n'est pas parce que l'on ne peut pas intervenir partout qu'il ne faut pas intervenir lorsqu'il est possible de le faire.

La sélectivité, inévitable et même souhaitable pour des raisons prudentielles, est un faux problème, mais elle a de vraies conséquences néfastes: elle attise la suspicion, la méfiance, le cynisme, nuit à la crédibilité des institutions et divise la supposée "communauté » internationale. Il faut donc la minimiser, agir de la façon la plus cohérente possible, mais sans aller jusqu'à sacrifier des populations entières - celles qu'on s'empêche de sauver quand ce serait possible - sur l'autel de la cohérence.

On peut utiliser ici la distinction utile faite par Peter Singer dans un autre domaine entre égalité de considération et égalité de traitement ${ }^{68}$. Il faut avoir une égalité de considération pour toutes les urgences humanitaires, quels que soient leur zone géographique, leur couverture médiatique, et les intérêts des uns et des autres. Il faut considérer également les intérêts de toutes les victimes: cette exigence dérive directement de l'universalité des droits humains. Mais il faut comprendre que cette égalité de considération n'implique pas forcément une égalité de traitement, c'est-à-dire que la réponse doit être adaptée à chaque cas. Considérer également des situations différentes peut - et même doit — aboutir à des traitements différents. D'une certaine manière, la consistance est donc satisfaite si l'égalité de considération est respectée. Le fait qu'elle n'implique pas une égalité de traitement est non seulement inévitable, mais aussi et pour plusieurs raisons souhaitables.

\section{Les droits humains dans la guerre (jus in bello)}

Jus in bello et jus ad bellum

Il y a d'abord un lien avec ce qui précède. D'un côté, faire la guerre au nom des droits humains (jus ad bellum) implique en principe une responsabilité spéciale de les respecter dans la guerre (jus in bello). Il ne faudrait pas se rendre coupable soi-même de ce contre quoi on intervient. De l'autre, faire la guerre au nom des droits humains donne une cause tellement "noble» et « urgente » qu'il pourrait être tentant de l'accomplir à n'importe quel prix, y compris en utilisant des moyens disproportionnés. Faisant référence aux armes modernes telles que les bombardiers et les avions d'attaque au sol,

68. En matière d'éthique animale: P. Singer, La libération animale, Paris, Grasset, I993, p. 3 I et 35 . 
Schmitt estimait en I950 qu' "il faut une guerre juste pour justifier l'emploi de tels moyens de destruction ${ }^{69}$ ".

C'est le risque de l'échelle mobile — une notion empruntée au monde du travail où elle signifie que les salaires s'adaptent automatiquement aux prix - de la conviction que " plus la guerre est juste, plus il y a de droits ${ }^{70}$ ". Or le DIH est le même pour tous les belligérants, pour ceux qui violent le droit comme pour ceux qui le respectent ${ }^{71}$. On peut alors défendre une double interaction entre jus ad bellum et jus in bello: non seulement la cause de l'intervention ne devrait pas justifier des violations du DIH, mais c'est même l'inverse: le respect du DIH par l'État intervenant au nom des droits humains devrait être pris en considération pour évaluer la légitimité — voire la légalité ${ }^{72}$ — de son intervention en matière de jus ad bellum ${ }^{73}$.

\section{DIH et DIDH}

Quoiqu'il en soit, la question des droits humains non plus comme cause de guerre mais dans la guerre est liée au DIH, qui est l'ensemble des règles cherchant à limiter les effets des conflits armés, dans le double objectif d'épargner celles et ceux qui ne participent pas (civils, personnel médical et religieux), ou plus (combattants blessés ou malades, naufragés, prisonniers de guerre) directement aux hostilités — c'est la protection des personnes (et de certains biens) - et de restreindre l'usage de la violence à la plus stricte nécessité militaire - c'est la restriction des moyens (armes) et méthodes (tactiques) de guerre. En tant que branche du droit international public, le DIH est relativement récent puisqu'il naît au milieu du XIX ${ }^{\mathrm{e}}$ siècle (avec la création du CICR en I 863, la première Convention de Genève en I 864, la Déclaration de Saint-Pétersbourg en I 868, etc.) mais, en tant qu'idéologie visant à humaniser la guerre, ses origines sont millénaires et correspondent à la partie jus in bello de la tradition de la guerre juste.

On s'accorde toutefois pour dire que les «droits humains " ne sont pas ceux protégés par le DIH, qui est un régime spécial propre au temps de guerre (lex specialis), mais par le DIDH, selon la division du travail expli-

69. C. Schmitt, Le Nomos de la terre, Paris, PUF, $200 \mathrm{I}$, p. 320.

70. M. Walzer, Guerres justes et injustes: argumentation morale avec exemples historiques, avec après-propos inédit de l'auteur, Paris, Gallimard, 2006, p. 4I3. Voir aussi R. Miller, "Legitimation, Justification, and the Politics of Rescue», in W. J. Buckley (ed.), Kosovo: Contending Voices on Balkan Interventions, Grand Rapids, William B. Eerdmans Publishing Company, 2000, p. 397-398.

71. H. Meyrowitz, Le principe de l'égalité des belligérants devant le droit de la guerre, Paris, Pedone, I970.

72. F. Mégret, "Jus in bello as jus ad bellum", in The Relationship Between Jus ad Bellum and Jus in Bello: Past, Present, Future, American Society of International Law (ASIL) Proceedings, 2006, p. I2I-I23.

73. E. Heinze, «Humanitarian Intervention and the War in Iraq : Norms, Discourse, and State Practice», Parameters, 36: I, 2006, p. 29. 
quée plus haut. Le DIDH étant applicable en tout temps (lex generalis), la question des droits humains dans la guerre est celle d'un chevauchement puisque, comme l'explique par exemple la Cour internationale de justice, «la protection offerte par le pacte international relatif aux droits civils et politiques ne cesse pas en temps de guerre ${ }^{74} »$. Ce problème qui donne lieu à une abondante littérature s'incarne par exemple dans les opérations de police ayant lieu en situation de conflit armé: sont-elles régies par le DIDH ou par le DIH? En d'autres termes, relèvent-elles des droits humains ou du droit de la guerre? Des droits humains, l'idée étant que ces opérations de police, parce qu'elles sont menées contre des civils, ne doivent pas être conduites de la même manière que des opérations (militaires) menées contre des combattants.

Les deux ont leurs différences. Dans l'approche des personnes protégées, d'abord: le DIH a des catégories de "personnes protégées", mais le DIDH est universaliste, il protège tous les individus sans distinction. Dans le rapport à l'État, ensuite: le DIH et le DIDH protègent tous les deux l'individu contre l'État (pour le DIH, contre l'État ennemi, ou belligérant dans tous les cas), mais le DIH le protège aussi contre les acteurs non étatiques (des groupes armés comme le Hamas, le Hezbollah, al-Qaïda, Daech, Boko Haram, etc.), édicte des règles de comportement pour les individus au bénéfice d'autres individus, et prévoit des sanctions individuelles (justice pénale internationale) - ce que ne fait pas le DIDH qui ne s'en prend qu'aux États. Autrement dit, le DIDH ne lie que les États (il ne crée pas d'obligations légales pour les groupes armés non étatiques et pour les individus), tandis que le DIH lie les États, les groupes armés non étatiques et les individus.

Une autre différence se trouve dans la distinction entre, d'une part, les droits civils et politiques et, d'autre part, les droits économiques, sociaux et culturels. Le DIDH fait cette distinction (dans les deux pactes de I966), même si elle est fréquemment critiquée (et sert à certains États à revendiquer leur «différence»). Le DIH ne fait pas de distinction: il protège des droits civils et politiques (comme le droit à la vie des ennemis hors de combat), des droits économiques, sociaux et culturels (comme le droit à la santé des habitants des territoires occupés, la protection de la propriété culturelle, etc.) et des droits collectifs (comme la protection de l'environnement).

Cette différence d'approche est problématique lorsque le DIH et le DIDH se chevauchent en situation de conflit armé mais ne font pas les mêmes recommandations. Le cas de figure est rare: dans la plupart des cas, les règles ne sont pas contradictoires. Mais, si cela arrive, lequel des deux prévaut? En principe, on applique le principe de la lex specialis (la règle spécifique prime sur la règle générale), qui est ancien puisque Grotius l'utili-

74. CIJ, Licéité de la menace ou de l'emploi d'armes nucléaires, avis consultatif du 8 juillet 1996 . 
sait déjà ${ }^{75}$. Mais ce principe d'interprétation, même s'il est majoritairement accepté, ne fait pas l'unanimité, et d'autres diront par exemple qu'il faut plutôt privilégier le droit qui fournit le degré de protection le plus élevé.

Deux exemples de conflits sont particulièrement évidents. D'une part, le recours à la force: le DIDH protège le droit à la vie, tandis que le DIH permet de tuer (pas n'importe qui et pas de n'importe quelle manière, mais tout de même). Le DIDH ne permet l'usage intentionnel de la force létale qu'en dernier recours et afin de protéger la vie, alors qu'en DIH, contre un combattant, il peut être un premier recours, et son but n'est pas nécessairement de protéger la vie. La contradiction est résolue en affirmant que la lex specialis prime, mais tout dépend du contexte, qui n'est pas toujours clair: les Américains par exemple estiment qu'ils sont parties à un conflit armé non international contre al-Qaïda «et ses forces associées » - d'où les éliminations ciblées au Pakistan, au Yémen et en Somalie. Cette interprétation extensive, car déterritorialisée, du conflit armé non international est-elle acceptable ?76

D'autre part, la détention: le DIH permet la détention de prisonniers de guerre et, dans certains cas, de civils (s'ils participent activement aux hostilités). L'État peut détenir ces personnes aussi longtemps que dure le conflit sans les juger: ce n'est donc pas une détention pénale (ce n'est pas une peine de prison), mais ce qu'on appellerait en temps de paix une détention (ou rétention) administrative (dont abusent certains États au nom de la lutte antiterroriste, contre l'immigration illégale, ou tout simplement pour se débarrasser d'opposants dans des dictatures). Mais ce qui est critiquable en temps de paix est accepté en temps de guerre. Là encore, de nombreuses zones grises suscitent des débats.

\section{Respect des droits et avantage stratégique ${ }^{77}$}

Le respect des droits en situation de conflit armé, qu'il s'agisse du DIH ou du DIDH, est parfois perçu comme un luxe, en tout cas pas comme une priorité, par des stratèges convaincus que l'efficacité militaire en souffrirait. Éthique et action stratégique sont généralement présentées comme antithétiques alors que, dans certains contextes, en particulier dans les situations de contre-insurrection qui sont désormais majoritaires, elles peuvent utilement converger.

Il y a des raisons strictement stratégiques de ne pas s'en prendre aux civils (ne pas s'aliéner ceux qui peuvent fournir des renseignements, voire, dans certaines situations, du ravitaillement, et ne pas les transformer en com-

75. Grotius, Droit de la guerre et de la paix, II, XVI, I9.

76. J.-B. Jeangène Vilmer, «Légalité et légitimité des drones armés », Politique étrangère, $20 \mathrm{I}_{3} / 3$, p. I I 9-I 32 et "Idéologie du drone", La vie des idées, 4 décembre 20I3, en ligne.

77. Voir J.-B. Jeangène Vilmer, «Éthique et action stratégique », in S. Taillat, J. Henrotin et O. Schmitt (dir.), Guerre et stratégie: approches, concepts, PUF, 20I 5, p. I7I-I 95. 
battants), et il y a aussi des raisons strictement éthiques et juridiques (respecter les principes du DIH: discrimination, humanité, précaution, etc.). Les deux coïncident dans la stratégie de gagner « les cœurs et les esprits». Comme le dit le Manuel de contre-insurrection de la US Army et des Marines, le soutien populaire est le «centre de gravité» des insurgés ${ }^{78}$. Une stratégie non éthique perdrait ce soutien populaire, et du même coup son efficacité.

Si la contre-insurrection française au Mali a mieux fonctionné que l'américaine en Afghanistan et en Irak, c'est parce qu'elle est basée sur la restriction de l'usage de la force et a réussi à obtenir le soutien des Maliens. La notion de force maîtrisée (l'un des "principes majeurs" du Code du soldat français) n'est pas seulement "opérative et tactique»: elle est aussi éthique $^{79}$. Le principe de maîtrise "n'est ni plus ni moins que le mode opératoire du principe d'humanitée ${ }^{80}$. Compte tenu de son importance en contexte contre-insurrectionnel, l'éthique militaire est vue par les armées comme ayant une valeur stratégique — d'où son enseignement dans les académies militaires.

Ainsi, l'avantage moral au sens du jus in bello (respect du DIH) procure un avantage stratégique. Et, a contrario, ce dont témoignent les abus de la «guerre contre la terreur » américaine est qu'avoir un désavantage moral (torture, Guantanamo, extraordinary rendition, etc.) procure un désavantage stratégique, en ruinant l'ethos américain et en entretenant la haine de l'Amérique (donc le risque d'attentats).

C'est ainsi qu'il faut défendre le respect des droits en temps de guerre: pas comme un principe déontologiste que les stratèges balaieront d'un revers de la main au nom de l'efficacité et de la nécessité militaire, mais comme une règle conséquentialiste qui produit généralement les meilleures conséquences pour tous les individus concernés, y compris ceux, civils et militaires, de la puissance intervenante.

\section{Conclusion: les droits humains après la guerre (jus post bellum)}

$\mathrm{Si}$, comme le rappelle Liddell Hart reprenant Clausewitz,

la guerre doit être la continuation de la politique, [...] elle doit être nécessairement conduite en ne perdant pas de vue le bénéfice de l'après-guerre. $[\ldots]$ L'objet de la guerre est d'obtenir une paix meilleure [...]. C'est pourquoi il est essentiel de conduire la guerre en ne perdant jamais de vue quelle paix vous souhaitez obtenir ${ }^{81}$.

78. The U.S. Army / Marine Corps, Counterinsurgency Field Manual, Chicago, The University of Chicago Press, 2007, p. IOI.

79. L'exercice du métier des armes dans l'armée de terre, fondements et principes, Étatmajor de l'armée de terre, Paris, janvier I999, p. I7.

80. J.-R. Bachelet, "La formation des militaires à l'éthique dans le métier des armes", Droits fondamentaux, 6, 2006, en ligne, p. 5 .

81. B. H. Liddell Hart, Stratégie, Paris, Perrin, 2007, p. 546 et 559. 
Ignorer les considérations éthiques - en l'occurrence le respect des droits humains - est une erreur stratégique, non seulement durant le conflit, car cela augmente le coût politique de la "victoire ", qui n'en est donc pas vraiment une, mais aussi après sa résolution, comme l'exemple libyen nous le montre cruellement.

On sait depuis longtemps, et plus encore dans les conflits asymétriques, qu'il est plus difficile de gagner la paix que de gagner la guerre. On sait depuis les années I990 que la fin des affrontements et la signature d'un accord de paix ne suffisent pas à garantir une paix durable. Environ la moitié des pays qui sortent d'un conflit majeur sombrent de nouveau dans un conflit dans les cinq ans qui suivent la signature d'un accord de paix. C'est pourquoi il a fallu ajouter une étape supplémentaire, après le rétablissement et le maintien de la paix, après la cessation des combats, après même la signature d'un accord, pour veiller à ce que la paix dure et que les conflits ne reprennent pas: c'est ce qu'on appelle la consolidation de la paix.

Définie dans l'Agenda pour la paix de Boutros-Ghali en I992, la consolidation de la paix implique

notamment de désarmer les adversaires, de rétablir l'ordre, de recueillir les armes et éventuellement de les détruire, de rapatrier les réfugiés, de fournir un appui consultatif et une formation au personnel de sécurité, de surveiller des élections, de soutenir les efforts de protection des droits de l'homme, de réformer ou de renforcer les institutions gouvernementales et de promouvoir des processus, formels ou informels, de participation politique ${ }^{82}$.

$\mathrm{Si}$, plus de vingt ans plus tard, ces objectifs peinent souvent à être mis en œuvre, c'est parce qu'il est objectivement très compliqué de le faire. On s'accorde par exemple sur trois volets: sécuritaire (désarmer, démobiliser, former de nouvelles forces de sécurité, réintégrer les anciens combattants), politique (promouvoir la démocratie - organisation d'élections et missions d'assistance électorale —, réformer la justice, défendre les droits humains) et économique et social (développer des infrastructures, faire des réformes économiques, etc.)

Mais dans ordre? Le volet sécuritaire semble prioritaire, car on ne peut pas mettre en place de nouvelles institutions, organiser des élections, faire des réformes, attirer des investisseurs étrangers et développer la vie économique s'il y a encore des problèmes de sécurité. En Angola, en I992, on n'a pas attendu de désarmer et de démobiliser pour organiser des élections présidentielles et législatives: elles ont contribué à la reprise de la guerre civile. Mais, en même temps, le volet sécuritaire dépend du volet politique, puisqu'on ne peut pas créer une nouvelle armée nationale sans la soumettre au contrôle d'un pouvoir politique fort, si l'on veut construire

82. B. Boutros-Ghali, Paix, développement, démocratie: trois agendas pour protéger la planète, Paris, Pedone, 2002, p. 22. 
une démocratie et rétablir la confiance dans l'armée. Il ne faut donc pas attendre pour réformer les institutions. Le paradoxe est alors le suivant: d'un côté, on ne peut pas mettre en place de nouvelles institutions s'il reste encore des problèmes de sécurité mais, de l'autre, pour les supprimer il faut mettre en place de nouvelles institutions.

Les volets sécuritaire et politique doivent donc être menés de front, avec également le volet économique et social car, pour faire tout ça, l'État a besoin de ressources financières. Si les moyens ne suivent pas, non seulement les réformes ne se feront pas, ou plus lentement, mais la paix elle-même peut être menacée. Voilà pourquoi la reconstruction est difficile. La «communauté internationale » peine en outre à être convaincue qu'une situation qui n'est plus une urgence mérite encore beaucoup d'attention.

La Libye a été victime de ces difficultés intrinsèques à toute reconstruction et d'un déficit d'attention dû à la diversion causée par le Mali et la Syrie. La «communauté internationale» a donc relativement échoué à mettre en œuvre le volet reconstruction de la $\mathrm{R}_{2} \mathrm{P}$ en Libye, on peut en tout cas lui reprocher de n'avoir pas fait suffisamment pour consolider la paix, et de sous-estimer aujourd'hui les dangers du chaos programmé qui s'annonce. Peut-on pour autant en conclure qu'elle a échoué dans sa responsabilité de protéger le peuple libyen? Je ne le crois pas, dans la mesure où elle l'a tout de même protégé de Kadhafi.

Bien sûr, les idéalistes disent qu'on a remplacé un mal par un autre, comme s'il avait été possible de ne pas le faire. Cette critique se fait sur l'horizon naif soit d'une non-intervention qui n'aurait pas été coupable de non-assistance à peuple en danger, soit d'une intervention qui aurait protégé le peuple libyen tout en transformant le pays en une démocratie stable du jour au lendemain. Le cas de la Libye n'est pas isolé: avec ou sans aide extérieure, les révolutions arabes depuis décembre 20Io n'ont pas accouché de situations stables, et l'avenir de plusieurs pays reste incertain. C'était prévisible car, comme le répète Hubert Védrine depuis longtemps, la démocratie n'est pas «du café instantané» mais un processus, qui a d'ailleurs mis des siècles à se construire en Occident.

À ceux qui disent qu'on a remplacé un mal par un autre, il faut donc demander lequel est le pire. Il est facile en effet de dire que c'est mal, comme si l'autre option était un bien qu'on se garde d'ailleurs de préciser. L'éthique réaliste que je défends ne pense pas en termes binaires de bien et de mal car, comme le disait Aron, "Ce n'est jamais la lutte entre le bien et le mal, c'est le préférable contre le détestable. Il en est toujours ainsi, en particulier en politique étrangère ${ }^{83}$. " L'éthique réaliste est celle du moindre mal ${ }^{84}$.

83. R. Aron, Le spectateur engagé, Paris, Julliard, I98I, p. 289-290.

84. J.-B. Jeangène Vilmer, "Pour un réalisme libéral en relations internationales ", Commentaire, I4I, printemps $20 \mathrm{I}_{3}$, p. I3-20. 